lymphoid tissue and alveolar macrophages. IPF patient lung section analysis revealed an absence of TRAIL expression compared to controls. IPF patients had significantly lower serum levels of TRAIL than controls which inversely correlated with TLCO (\% predicted) and positively correlated with survival from diagnosis.

Conclusions We demonstrated that the neutrophilic inflammatory response to bleomycin is increased in TRAIL-/- compared with wild-type mice and that this finding is associated with increased collagen deposition. We also demonstrated reduced pulmonary and systemic expression of TRAIL in IPF, which correlates with worse pulmonary function and clinical outcome. This data suggests TRAIL may have biomarker potential and therapeutic benefit in pulmonary fibrosis.

\section{S112 TNF-R1 UBIQUITOUS SCAFFOLDING AND SIGNALLING PROTEIN (TRUSS) IS A REGULATOR OF TNF- $\alpha$ INDUCED NF-K-B ACTIVATION}

doi:10.1136/thoraxjnl-2011-201054b.112

${ }^{1} \mathrm{~A} J$ Langton, ${ }^{1} \mathrm{I}$ Horan, ${ }^{2} \mathrm{~S}$ Farrow, ${ }^{3} \mathrm{D}$ W Riches, ${ }^{1} \mathrm{E}$ R Chilvers, ${ }^{1} \mathrm{H}$ Parfrey. ${ }^{1}$ University of Cambridge, Cambridge, UK; ${ }^{2}$ GlaxoSmithKline, Stevenage, UK; ${ }^{3}$ National Jewish Health, Denver, USA

The pleiotropic cytokine TNF $\alpha$ plays a key role in the pathogenesis of many chronic inflammatory lung diseases, particularly sarcoidosis, asthma and COPD. Due to its broad spectrum of activity however, current anti-TNF $\alpha$ therapies are of limited efficacy in these conditions and are associated with an increased risk of infection and malignancy. Interaction of TNF $\alpha$ with its cognate receptor, TNF-R1 initiates a signalling cascade that leads ultimately to the phosphorylation of the transcription factor NF-kB. This allows NF-kB to shuttle in a co-ordinated manner between the cytoplasm and the nucleus, leading to the up-regulation of genes that are key to cellular inflammatory and apoptotic responses. We propose that the novel TNF-R1 interacting protein TRUSS (TNF-R1 Ubiquitous Scaffolding and Signalling protein), which interacts with members of the TNFR1 signalling cascade, may regulate this process. A549 cells, which express high levels of endogenous TRUSS, were transfected transiently with siRNA, which resulted in $80 \pm 14 \%$ (mean \pm SEM, $n=16$ ) knockdown of TRUSS mRNA. TRUSS deficient cells demonstrated a profound early $(<1 \mathrm{~h})$ defect in the nuclear translocation of $\mathrm{p} 50 / \mathrm{p} 65$ subunits following TNF $\alpha$ stimulation $(p<0.05, n=3)$. As a consequence, in the absence of TRUSS, p50, its precursor phospho-p105, and phospho-p65 were retained in the cytoplasm in these cells following TNF $\alpha$ stimulation. Furthermore, TRUSS depletion caused a reduction in TNF $\alpha$ stimulated NF-kB $(p<0.01, n=7)$ and AP-1 $(p<0.01, n=6)$ luciferase reporter activity; this was associated with a decrease in interleukin 6, RANTES, G-CSF and GM-CSF $(p<0.05$, $\mathrm{n}=6)$ mRNA and protein expression while MCP-1, CXCL5 and IL-8 were not affected. Although TRUSS deplete cells displayed impaired up-regulation of IkBa mRNA in response to TNF $\alpha$ stimulation, the protein response was intact. Upstream signalling molecules TNFR1, TRADD, TRAF2 and RIP were unaffected by TRUSS knockdown. In conclusion, these data suggest a novel role for TRUSS as a scaffold protein involved in the initial nuclear translocation of $\mathrm{p} 50 / \mathrm{p} 65$ NF-kB subunits, which regulates the early pro-inflammatory response to TNF $\alpha$. Hence TRUSS may represent a more selective therapeutic target for modulating TNF $\alpha$ functions.

\section{S113 THE INFLUENZA VIRUS ACTIVATES TGFß VIA AN $\alpha$ VB6- INTEGRIN MEDIATED PATHWAY}

doi:10.1136/thoraxjnl-2011-201054b.113

${ }^{1}$ A S Stavrou, ' $\mathrm{L}$ J Jolly, ${ }^{1} \mathrm{~J}$ P Porte, ${ }^{2} \mathrm{P}$ W Weinreb, ${ }^{1} \mathrm{~A}$ T Tatler, ${ }^{1} \mathrm{~A}$ J John, ${ }^{1} \mathrm{~A}$ K Knox, ${ }^{2} \mathrm{~S}$ V Violet, ${ }^{3} \mathrm{~T}$ H Hussel, ' $\mathrm{S}$ K Kuchipudi, ${ }^{1} \mathrm{~S} D$ Dunham, ${ }^{1} \mathrm{G}$ J Jenkins. ${ }^{1}$ University of

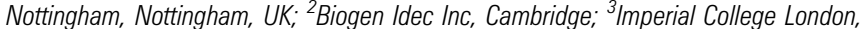
London, UK

Introduction and Objectives Idiopathic pulmonary fibrosis is a chronic progressive lung disease of unknown cause. Its pathogenesis is poorly understood but activation of latent TGFß on lung epithelium is an important factor. TGFß must be activated, as it is secreted in a latent complex with its propeptide, the latency associated peptide, and the avß6 integrin is a key activator in the lung. The Influenza $A$ virus is a single-stranded segmented RNA virus that infects epithelial cells leading to cell death and injury. Toll-like receptors (TLRs) detect pathogens, such as influenza. TLR3 activation has been found to increase RhoA activity. We previously showed that RhoA is a key intermediary in avß6 integrin-mediated TGFß activation. The aim of this study is to investigate whether influenza can activate TGFß and stimulate TLR3 leading to activation of TGFß through the avß6 integrin in epithelial cells.

Materials and Methods Immortalised human bronchial epithelial cells (iHBECs) were infected with influenza A ( $\mathrm{H} 2 \mathrm{~N} 3)$ virus at a multiplicity of infection 1 with, or without, the avß6 blocking antibody 6.3G9. iHBECs were also stimulated with the synthetic TLR3 ligand poly(I:C). TGFß activity was determined by: (1) immunoblotting for phosphorylated (phospho-) Smad2, and (2) Transformed mink lung cells (TMLC)-iHBEC cocultures. Infection efficiency was measured by Interferon $B$ mRNA levels by real-time qPCR.

Results Infection with $\mathrm{H} 2 \mathrm{~N} 3$ and stimulation with poly(I:C) led to increase in phospho-smad2 and luciferase activity in coculture indicating increase in TGFß activation levels in a dose- and timedependent manner. In both cases this was blocked with the addition of 6.3G9. qPCR data following infection showed increased IFNB1 and PAI-1, indicating the ability of the virus to infect the cells and activate TGFß.

Conclusions Influenza infection and poly(I:C) activates TGFß in iHBECs in an avß6 integrin dependent manner. The data suggests a novel mechanism by which influenza infection of epithelial cells may promote lung fibrosis.

\section{S114 LYMPHATIC VESSEL DISTRIBUTION IN FIBROTIC LUNG DISEASES}

doi:10.1136/thoraxjnl-2011-201054b.114

${ }^{1}$ E A Renzoni, ${ }^{2}$ E Weber, ${ }^{2}$ F Sozio, ${ }^{2}$ A Rossi, ${ }^{1}$ A U Wells. ${ }^{1}$ Interstitial Lung Disease Unit, Royal Brompton Hospital, London, UK; ${ }^{2}$ Molecular Medicine Section, Department of Neuroscience, University of Siena, Siena, Italy

Relatively little is known on lymphatic vessel remodelling in lung fibrosis, and whether differences in lymphatic distribution underlie the worse survival seen in idiopathic pulmonary fibrosis, compared to the other fibrotic interstitial pneumonias. Lymphatic vessel remodelling and a deficit in lymphatic clearance could lead to prolonged exposure to pathogenic antigens and/or pro-inflammatory/pro-fibrotic mediators in the alveolar-interstitial space. In this study, we compared lymphatic and blood vessel morphology in lung biopsies of six patients with idiopathic pulmonary fibrosis (with usual interstitial pneumonia pattern-UIP), six patients with fibrotic non-specific interstitial pneumonia associated with scleroderma (NSIP) five patients with fibrotic organising pneumonia (FOP) and five controls (normal lung peripheral to resected cancer). Consecutive sections were stained with Movat's pentachrome and with double immunostaining for von Willebrand factor (blood vessels) and for podoplanin (lymphatic specific marker D2-40) (see Abstract S114 figure 1). Area, perimeter and position were recorded for all lymphatic vessels and for blood vessels with a diameter $>15 \mu \mathrm{m}$, to be sure to exclude capillaries. In the three disease groups, blood 